\title{
Newton Polygons and Formal Gevrey Classes
}

\author{
By
}

\author{
Akiyoshi YonemurA*
}

\section{Introduction}

Following to the fundamental study of Malgrange [7], Ramis elucidated the analytic meaning of slope of Newton polygon for ordinary differential operators [10]: In generic cases the index of operator in formal Gevrey class of order $s$ equals to the ordinate at the origin of supporting line of Newton polygon with slope $k=1 /(s-1)$. $\mathrm{He}$ also demonstrated various comparison theorems.

The purpose of this note is to generalize one aspect of Ramis theory to partial differential operators. There seems to be three ways of generalization:

1. To consider holonomic systems.

2. To consider operators of Kashiwara-Kawai-Sjöstrand type [1, 3].

3. To consider Cauchy problems.

For 1, 2, we refer to Laurent theory $[4,5,6]$. We shall discuss from the standpoint 3.

On the other hand, our study is closely related to the Cauchy-Kowalewski theorem. Mizohata's inverse Cauchy-Kowalewski theorem asserts that if the operator is not Kowalewskian, there exists a divergent formal solution [8]. It is well known that the formal solution of heat equation belongs to Gevrey class of order 2. The problem is what determines the Gevrey order of formal solutions.

From a different point of view, Ouchi developed the theory concerning the analytic meaning of formal solutions [9]. It is certain that his theory implies one part of our theorem. There exists, however, more elementary and straightforward method to our problem.

\section{§1. Notations}

For $x=\left(x_{1}, x_{2}, \ldots, x_{n}\right) \in \mathbb{C}^{n}$, we set $|x|=\max _{1 \leq j \leq n}\left|x_{j}\right| . \quad$ Let $\mathcal{O}(|x|<r)$ be the set of all holomorphic functions in $\left\{x \in \mathbb{C}^{n} ;|x|<r\right\}$. We also set

$$
\mathcal{O}(|x| \leq r)=\mathscr{C}^{0}(|x| \leq r) \cap \mathcal{O}(|x|<r)
$$

where $\mathscr{C}^{0}(|x| \leq r)$ is the set of all continuous functions on $\left\{x \in \mathbb{C}^{n} ;|x| \leq r\right\}$.

Communicated by S. Matsuura, March 22, 1989.

* Faculty of Engineering, Osaka Institute of Technology, Asahi-ku, Osaka, 535, Japan. 
It is obvious that $\mathcal{O}(|x| \leq r)$ is a Banach space with maximum norm $\|\cdot\|_{r}$.

Let $\mathbb{C}[[t, x]]$ be the set of formal power series with complex coefficients in $n+1$ indeterminates $t, x$. Let $\mathbb{C}\{t, x\}$ be the set of convergent power series in $n+1$ variables $(t, x)=\left(t, x_{1}, \ldots, x_{n}\right)$. When we set $A=\mathcal{O}(|x| \leq r)$ or $\mathbb{C}\{x\}$, we denote by $A[[t]]$ the set of formal power series in $t$ with coefficients in $A$. These are subspaces of $\mathbb{C}[[t, x]]$.

We shall use standard multi-indices notations:

$$
\begin{aligned}
& D_{t}=\frac{\partial}{\partial t}, \quad D_{j}=\frac{\partial}{\partial x_{j}} \quad(j=1,2, \ldots, n), \\
& D_{x}^{\alpha}=D_{1}^{\alpha_{1}} \ldots D_{n}^{\alpha_{n}} \quad \text { for } \quad \alpha \in \mathbb{N}^{n} .
\end{aligned}
$$

\section{§2. Definitions}

Let $P$ be a differential operator with coefficients $\in \mathbb{C}[[t, x]]$ :

$$
P=P\left(t, x ; D_{t}, D_{x}\right)=\sum_{j, \alpha} a_{j, \alpha}(t, x) D_{t}^{j} D_{x}^{\alpha}=\sum_{j, \alpha} t^{\sigma(j, \alpha)} \tilde{a}_{j, \alpha}(t, x) D_{t}^{j} D_{x}^{\alpha}
$$

where $\tilde{a}_{j, \alpha}(0, x) \neq 0$ in $\mathbb{C}[[x]]$. Let $Q$ be the second quadrant of $\mathbb{R}^{2}$ and for $(u, v) \in \mathbb{R}^{2}$, we set

$$
Q(u, v)=(u, v)+Q
$$

Definition. The Newton polygon of $P$, denoted by $N(P)$, is defined by the convex hull of the union of $Q(j+|\alpha|, \sigma(j, \alpha)-j)$ for $j, \alpha$ such that $a_{j, \alpha} \neq 0$ in $\mathbb{C}[[t, x]]$ :

$$
N(P)=\operatorname{ch}\left(\bigcup_{a_{j, \alpha} \neq 0} Q(j+|\alpha|, \sigma(j, \alpha)-j)\right) .
$$

Let $0=k_{0}<k_{1}<\cdots<k_{l}$ be the slopes of sides of $N(P)$.

Remark. If $P$ is a differential operator with holomorphic coefficients, this definition is a special case of more general one $[4,5,6]$ : If we choose

$$
X=\mathbb{C}^{n+1}=\mathbb{C}_{t} \times \mathbb{C}_{x}^{n}, Y=\{t=0\} \subset X, \Lambda=T_{Y}^{*} X \text { and } O=(o ; o) \in X,
$$

then according to Laurent's notation [5] we have

$$
N(P)=N_{\Lambda, o}(P) .
$$

Let us notice that this definition is different from that of Mizohata [8]. For example, it suffices to consider the operator $P=D_{t}^{2}+D_{t} D_{x}^{2}+t^{2} D_{x}^{5}$.

To examine the analytic meaning of $k_{j}$, we define the functions of formal Gevrey class.

Definition. Let $s \geq 1, \rho>0$ and $r>0$. Then we denoted by $G_{\rho, r}^{s}$ the set of all $u=\sum_{j=0}^{\infty} u_{j} t^{j} \in \mathcal{O}(|x| \leq r)[[t]]$ such that

$$
|u|_{\rho, r}^{s} \stackrel{\text { def }}{=} \sum_{j=0}^{\infty} \frac{\left\|u_{j}\right\|_{r}}{(j !)^{s-1}} \rho^{j}<+\infty .
$$


Lemma 1. $G_{\rho, r}^{s}$ is a Banach space with norm $|\cdot|_{\rho, r}^{s}$.

The proof is obvious.

We set

$$
G_{\rho}^{s}=\bigcup_{r>0} G_{\rho, r}^{s} \text { and } G^{s}=\bigcup_{\rho>0} G_{\rho}^{s} .
$$

Note that $G^{1}=\mathbb{C}\{t, x\}$. If we also set $G^{\infty}=\mathbb{C}\{x\}[[t]]$, then we have interpolation spaces $G^{s}$ between the space of convergent power series and that of formal power series: for $1<s<\infty$,

$$
\mathbb{C}\{t, x\}=G^{1} \subset G^{s} \subset G^{\infty}=\mathbb{C}\{x\}[[t]] \subset \mathbb{C}[[t, x]] .
$$

\section{§3. Statement of Theorem}

Let $P$ be a differential operator of the following form:

$$
P=D_{t}^{m}+\sum_{0 \leq j<m} a_{j, \alpha}(t, x) D_{t}^{j} D_{x}^{\alpha},
$$

where $a_{j, \alpha} \in G^{s}$. We assume that $P$ is not Kowalewskian:

$$
\text { ord } P>m \text {. }
$$

We consider the Cauchy problem

$$
(C P)\left\{\begin{array}{l}
P u=f(t, x) \\
\left.D_{t}^{j} u\right|_{t=0}=g_{j}
\end{array} \quad \text { for } \quad 0 \leq j \leq m-1\right.
$$

where

$$
f \in G^{s}, g_{j} \in \mathbf{C}\{x\} .
$$

There exists a unique formal solution $u \in G^{\infty}$. The Cauchy-Kowalewski theorem asserts that, if $P$ is Kowalewskian, $u$ is convergent. We investigate precisely the relation between the divergence order of $u$ and the Newton polygon of $P$.

Theorem 1. Let $s=1+1 / k_{1}$. Then there exists a unique solution $u \in G^{s}$, satisfying $(C P)$.

Remark 1. Particularly for $f, a_{j, \alpha} \in \mathbf{C}\{t, x\}$, a fortiori the assertion of theorem holds. We rediscover one corollary of Ōuchi's results [9].

Remark 2. This result is best possible: In general one cannot lower the Gevrey order $s$. For example, let

$$
n=1, \quad P=D_{t}-t^{\sigma} D_{x}^{m}, \quad f=0 \quad \text { and } \quad g=\sum_{j=0}^{\infty} x^{j} \in \mathcal{O}(|x|<1),
$$

where $\sigma \in N, m \geq 2$. Then we have

$$
u=\sum_{i, j \geq 0} \frac{(m i+j) !}{(\sigma+1)^{i} i ! j !} t^{(\sigma+1) i} x^{j}, \quad k_{1}=\frac{\sigma+1}{m-1} \quad \text { and } \quad s_{1}=\frac{\sigma+m}{\sigma+1} .
$$


It follows that

$$
u \in G^{s} \text { for } s \geq s_{1} \text {, but } u \notin G^{s} \text { for } s<s_{1} \text {. }
$$

\section{§4. Formal Norm and Lemmas}

For $u \in G_{\rho, r}^{s}$, we shall use the formal norm:

$$
N_{r}^{s}[u](t) \stackrel{\text { def }}{=} \sum_{j=0}^{\infty} \frac{\left\|u_{j}\right\|_{r}}{(j !)^{s-1}} t^{j}
$$

If $|t| \leq \rho$, then we have

$$
\left|N_{r}^{s}[u](t)\right| \leq|u|_{\rho, r}^{s}, \quad N_{r}^{s}[u](\rho)=|u|_{\rho, r}^{s} .
$$

We set

$$
\left(D_{t}^{-1} u\right)(t)=\sum_{j=0}^{\infty} u_{j} \frac{t^{j+1}}{j+1} \quad \text { for } \quad u \in \mathcal{O}(|x| \leq r)[[t]] .
$$

Lemma 2。 Let $a, u \in G_{\rho, r}^{s}$. The following properties hold for $0 \leq t \leq \rho$ :

$$
\begin{gathered}
N_{r}^{s}[a u](t) \leq N_{r}^{s}[a](t) \cdot N_{r}^{s}[u](t) \\
N_{r^{\prime}}^{s}\left[D_{i} u\right](t) \leq \frac{1}{r-r^{\prime}} N_{r}^{s}[u](t)
\end{gathered}
$$

for $0<r^{\prime}<r, i=1,2, \ldots, n$.

The proof is straightforward. Inequality (1) asserts that $G_{\rho, r}^{s}$ is a Banach algebra. Notice that in general $D_{t}$ nor $D_{i}$ do not operate on $G_{\rho, r}^{s}$.

We detine the operators $A_{s}, B_{s}$ acting on $\mathbb{R}\{t\}$ :

$$
\begin{gathered}
N_{r}^{s}\left[D_{t}^{-1} u\right](t)=A_{s}\left(N_{r}^{s}[u]\right)(t) \\
A_{s}: \sum c_{j} t^{j} \mapsto \sum c_{j} \frac{t^{j+1}}{(j+1)^{s}} \\
N_{r}^{s}[t u](t)=B_{s}\left(N_{r}^{s}[u]\right)(t) \\
B_{s}: \sum c_{j} t^{j} \mapsto \sum c_{j} \frac{t^{j+1}}{(j+1)^{s-1}}
\end{gathered}
$$

(4) where

(6) where

Proposition 1. Let $T$ and $s$ be non-negative real numbers. Let $f(t)=\sum_{j=0}^{\infty} c_{j} t^{j} \in$ $\mathbb{R}\{t\}$ with radius of convergence $>T$. If $f(t) \geq 0$ for $0 \leq t \leq T$, then

$$
\left(L_{s} f\right)(t) \stackrel{\operatorname{def}}{=} \sum_{j=0}^{\infty} c_{j} \frac{t^{j}}{(j+1)^{s}} \geq 0
$$

for $0 \leq t \leq T$.

Since the assertion is trivial for $s=0$, we assume $s>0$. It suffices to prove that $L_{s}$ has the following integral representation: for $f$ stated above, 


$$
\left(L_{s} f\right)(t)=\frac{1}{\Gamma(s)} \int_{0}^{\infty} e^{-\tau} \tau^{s-1} f\left(t e^{-\tau}\right) d \tau .
$$

The convergence of integral is proved in the same way as that of Euler's expression of Gamma-function. For $f(t)=t^{n}$, we have

$$
\begin{aligned}
\frac{1}{\Gamma(s)} \int_{0}^{\infty} e^{-\tau} \tau^{s-1}\left(t e^{-\tau}\right)^{n} d \tau & =\frac{t^{n}}{\Gamma(s)} \int_{0}^{\infty} e^{-(n+1) \tau} \tau^{s-1} d \tau \\
& =\frac{t^{n}}{(n+1)^{s}} \frac{1}{\Gamma(s)} \int_{0}^{\infty} e^{-\tau} \tau^{s-1} d \tau \\
& =\frac{t^{n}}{(n+1)^{s}} .
\end{aligned}
$$

This implies that (7) holds for $f$ polynomial. The right side of (7) is a continuous operator in $\mathscr{C}^{0}[0, T]$ and $f_{n}=\sum_{j=0}^{j=n} c_{j} t^{j}$ converges to $f$ in $\mathscr{C}^{0}[0, T]$. In addition $L_{s}\left(f-f_{n}\right) \rightarrow 0(n \rightarrow \infty)$ in $\mathscr{C}^{0}[0, T]$ by the fact that Taylor series are absolutely and uniformly convergent on any compact subset in the circle of convergence. Thus (7) holds for any $f$ stated above.

Since we have $A_{s-1}=B_{s}, A_{s} f=t\left(L_{s} f\right)(t)$, the proposition means that operators $A_{s}$, $B_{s}$ preserve inequalities.

\section{§5. Proof of Theorem 1}

First we show that the assumption $s=1+1 / k_{1}$ implies that

$$
|\alpha| \leq(s-1) \sigma(j, \alpha)+s(m-j) .
$$

Indeed, Newton polygon of $P$ has both vertex $(m,-m)$ and side of slope $k_{1}$ through $(m,-m)$. Since the points $(j+|\alpha|, \sigma(j, \alpha)-j)$ are included in the upper half plane defined by $y \geq k_{1}(x-m)-m$, we obtain

$$
\sigma(j, \alpha)-j \geq k_{1}(j+|\alpha|)-\left(k_{1}+1\right) m \Longleftrightarrow|\alpha| \leq \frac{1}{k_{1}} \sigma(j, \alpha)+\left(1+\frac{1}{k_{1}}\right)(m-j),
$$

which proves (8).

Let $P=D_{t}^{m}-Q$ where

$$
Q=-\sum_{j=0}^{m-1} \tilde{a}_{j, \alpha} D_{x}^{\alpha} t^{\sigma(j, \alpha)} D_{t}^{j}
$$

We define a sequence $\left\{u_{k}\right\}$ as follows:

$$
\left\{\begin{array}{l}
D_{t}^{m} u_{0}=f \\
\left.D_{t}^{j} u_{0}\right|_{t=0}=g_{j} \quad(0 \leq j \leq m-1) .
\end{array}\right.
$$

For $k \geq 0$,

$$
\left\{\begin{array}{l}
D_{t}^{m} u_{k+1}=Q u_{k}+f \\
\left.D_{t}^{j} u_{k+1}\right|_{t=0}=g_{j} \quad(0 \leq j \leq m-1)
\end{array}\right.
$$


Next we set

$$
\begin{aligned}
v_{0} & =u_{0} \\
v_{k+1} & =u_{k+1}-u_{k} \quad \text { if } \quad k \geq 0
\end{aligned}
$$

Then we have for $k \geq 1$,

$$
\left\{\begin{array}{l}
D_{t}^{m} v_{k}=Q v_{k-1}, \\
\left.\mathrm{D}_{t}^{j} v_{k}\right|_{t=0}=0 \quad(0 \leq j \leq m-1) .
\end{array}\right.
$$

We also set $w_{k}=D_{t}^{m} v_{k}$, then we have for $k \geq 1, v_{k}=D_{t}^{-m} w_{k}$. Then the sequence $\left\{w_{k}\right\}$ satifies the following equation:

$$
\begin{gathered}
w_{0}=D_{t}^{m} u_{0}=f, \\
w_{k+1}=Q D_{t}^{-m} w_{k} \quad(k \geq 0)
\end{gathered}
$$

where

$$
Q D_{t}^{-m}=\sum_{0 \leq j<m, \alpha} \tilde{a}_{j, \alpha} D_{x}^{\alpha} t^{\sigma(j, \alpha)} D_{t}^{-(m-j)} w_{k} .
$$

Let $T$ and $r_{0}$ be positive real numbers such that $f, \tilde{a}_{j, \alpha} \in G_{T, r_{0}}^{s}$. We fix $\left.r_{1} \in\right] 0, r_{0}[$. It follows immediately that for $0<\rho<T$ and $0<r<r_{0}$,

$$
u_{k}, v_{k}, w_{k} \in G_{\rho, r}^{s} .
$$

Let $K$ and $M$ denote positive constants such that

$$
N_{r_{0}}^{s}[f](T)=K \text { and } N_{r_{0}}^{s}\left[\tilde{a}_{j, \alpha}\right](T) \leq M
$$

for any $\tilde{a}_{j, \alpha}$ which appears in $P$. We prove the following inequality by induction on $k$ : There exist a positive constant $C$ such that for $k \in \mathbb{N}$ and $r \in] r_{1}, r_{0}[$,

$$
N_{r}^{s}\left[w_{k}\right] \leq K C^{k} \frac{e^{d k} t^{k}}{\left(r_{0}-r\right)^{d k}}
$$

where $d=\max \left\{|\alpha| ; a_{j, \alpha} \neq 0\right\}$.

Let us take $r \in] r_{1}, r_{0}\left[\right.$ and $r^{\prime}>r$. From (1), (2), (9), we have

$$
\begin{aligned}
N_{r}^{s}\left[w_{k+1}\right] & \leq \sum \frac{M}{\left(r^{\prime}-r\right)^{|\alpha|}} N_{r^{\prime}}^{s}\left[t^{\sigma(j, \alpha)} D_{t}^{-(m-j)} w_{k}\right] \\
& =\sum \frac{M}{\left(r^{\prime}-r\right)^{|\alpha|}}\left(B_{s}^{\sigma(j, \alpha)} A_{s}^{m-j}\right) N_{r^{\prime}}^{s}\left[w_{k}\right] \\
& =\sum \frac{M}{\left(r^{\prime}-r\right)^{|\alpha|}}\left(B_{s}^{v(j, \alpha)} A_{s}^{j}\right) N_{r^{\prime}}^{s}\left[w_{k}\right]
\end{aligned}
$$

where we set $v(j, \alpha)=\sigma(m-j, \alpha)$ for $1 \leq j \leq m$. Then from (8), we have

$$
|\alpha| \leq(s-1) v(j, \alpha)+s j .
$$

If we assume that (10) holds for $k$, we get from Proposition 1 and (11), 


$$
N_{r}^{s}\left[w_{k+1}\right] \leq K M C^{k} e^{d k} \sum \frac{1}{\left(r^{\prime}-r\right)^{|\alpha|}\left(r_{0}-r^{\prime}\right)^{d k}}\left(B^{v(j, \alpha)} A^{j}\right)\left[t^{k}\right] .
$$

We now choose $r^{\prime}=r+\left(r_{0}-r\right) /(k+1)$, so that $r_{0}-r^{\prime}=\left(r_{0}-r\right) /(1+1 / k)$. Then for the coefficients of $t^{k+j+v(j, \alpha)}$ under sigma sign, we have

$$
\begin{gathered}
\frac{1}{\left(r^{\prime}-r\right)^{|\alpha|}\left(r_{0}-r^{\prime}\right)^{d k}} \frac{1}{((k+1) \ldots(k+j))^{s}\left((k+j+1) \ldots(k+j+v(j, \alpha))^{s-1}\right.} \\
=\frac{(1+1 / k)^{k d}}{\left(r_{0}-r\right)^{|\alpha|+d k}} \frac{(k+1)^{|\alpha|}}{((k+1) \ldots(k+j))^{s}\left((k+j+1) \ldots(k+j+v(j, \alpha))^{s-1}\right.}
\end{gathered}
$$

By (12), the second fraction is less than or equal to

$$
\left(\frac{(k+1)^{j}}{(k+1) \ldots(k+j)}\right)^{s}\left(\frac{(k+1)^{v(j, \alpha)}}{(k+j+1) \ldots(k+j+v(j, \alpha))}\right)^{s-1},
$$

which is less than or equal to 1 . Thus we obtain

$$
N_{r}^{s}\left[w_{k+1}\right] \leq K C^{k} \frac{e^{d(k+1)} t^{k+1}}{\left(r_{0}-r\right)^{d(k+1)}} M \sum_{j \geq 1, \alpha}\left(r_{0}-r\right)^{d-|\alpha|} t^{j-1+v(j, \alpha)} .
$$

It suffices to take the constant $C$ by

$$
C=M \sum_{j \geq 1, \alpha}\left(r_{0}-r_{1}\right)^{d-|\alpha|} T^{j-1+v(j, \alpha)}
$$

If we choose $\varepsilon \in] 0, T]$ such that

$$
\frac{C e^{d} \varepsilon}{\left(r_{0}-r\right)^{d}}<1
$$

it follows from (10) that $\sum_{k=0}^{\infty} w_{k}$ is convergent in $G_{\varepsilon, r}^{s}$. Since $D_{t}^{-m}$ is a continuous operator in $G_{\varepsilon, \mathrm{r}}^{s}$ and that $D_{t}^{m}, Q: G_{\varepsilon, r}^{s} \rightarrow G_{\varepsilon_{1}, r_{1}}^{s}$ are continuous operators for $\left.\varepsilon_{1} \in\right] 0, \varepsilon[$, it follows that

$$
u=\lim _{k \rightarrow \infty} u_{k}=\sum_{k=0}^{\infty} v_{k} \in G_{\varepsilon, r}^{s} \subset G_{\varepsilon_{1}, r_{1}}^{s}
$$

and $u$ satisfies $(C P)$ in $G_{\varepsilon_{1}, r_{1}}^{s}$. The proof is complete.

\section{§6. Further Generalizations}

To make the assertions clear, we stated Theorem 1 under more restrictive assumptions, which we shall make less strict as follows.

1. Theorem 1 also holds for operators of the following type:

$$
P=\sum_{j, \alpha} a_{j, \alpha}(t, x) D_{t}^{j} D_{x}^{\alpha}
$$

where $a_{m, 0}(t, x)$ is a unit in $\mathbb{C}[[t, x]]$ and the point $(m,-m)$ is a vertex of $N(P)$. Notice that in this case order of $P$ with respect to $D_{t}$ may be larger than $m$.

2. For $P$, we denote its principal part by 


$$
\sigma(P)=\sum^{\prime} a_{j, \alpha} D_{t}^{j} D_{x}^{\alpha}
$$

where $\sum^{\prime}$ means that sum is taken for all $(j, \alpha)$ such that $\sigma(j, \alpha)-j=\min [\sigma(j, \alpha)-j]$, namely sum of the terms of $P$ which correspond to the points lying on the side of $N(P)$ parallel to abscissa. The operators discussed so far have the term $D_{t}^{m}$ as principal part.

Theorem 2. The assertion of Theorem 1 also holds for operators $\mathbb{P}$ such that $\sigma(\mathbb{P})$ is Fuchsian in the sense of Baouendi-Goulaouic under the usual conditions on characteristic exponents [2].

Needless to say we have to modify the number of Cauchy data in this case.

These assertions are proved in the same way as Theorem 1.

Acknowledgement. I would like to thank the referee for his critical reading the manuscript and useful comments. Especially, I owe to him the example in Remark 2, section 3.

\section{References}

[1] Aoki, T., Kashiwara, M. and Kawai, T., On a class of linear differential operators of infinite order with finite index, Adv. in Math. 62 (1986), 152-168.

[2] Baouendi, M. S. and Goulaouic, C., Cauchy problems with characteristic initial hypersurface, Comm. Pure App. Math. 2 (1973), 455-475.

[3] Kashiwara, M., Kawai, T. and Sjöstrand, J., On a class of linear partial differential equations whose formal solutions always converge, Ark. Mat. 17 (1979), 83-91.

[4] Laurent, Y., Théorie de la Deuxième Microlocalisation dans le Domaine Complexe, Progress in Math. 53, Birkhäuser, 1985.

[5] Calcul d'induces et irrégularité pour les systèmes holonômes, Astérisque 130 (1985), $352-364$.

[6] —, Polygône de Newton et b-foctions pour les modules micro-différentiels, Ann. Sci. École Norm. Sup. 20 (1987), 391-441.

[7] Malgrange, B., Sur les points singuliers des équations différentielles, Enseign. Math. 20 (1974), 147176.

[8] Mizohata, S., On the Cauchy-Kowalewski theorem, Mathematical Analysis and Applications, Part B; Adv. in Math. Supplementary Studies, 7B (1981), 617-652.

[9] Öuchi, S., Characteristic Cauchy problems and solutions of formal power series, Ann. Inst. Fourier (Grenoble) 33 (1983), 131-176.

[10] Ramis, J.-P., Théorèmes d'indices Gevrey pour les équations différentielles ordinaires, Memoirs of the Am. Math. Soc. Vol. 48, No. 296, 1984.

[11] Treves, F., Basic Linear Partial Differential Equations, Academic Press, 1975. 